Nedela, Daniel. Análisis de la presencia de las multilatinas en el espacio económico del grupo de Visegrád.

\title{
ANÁLISIS DE LA PRESENCIA DE LAS MULTILATINAS EN EL ESPACIO ECONÓMICO DEL GRUPO DE VISEGRÁD
}

\author{
DANIEL NEDELA \\ Universidad Complutense de Madrid \\ danielnedela89@gmail.com
}

Fecha de recepción: septiembre de 2015

Fecha de aceptación: diciembre de 2015

\begin{abstract}
RESUMEN
Este trabajo pretende ampliar la todavía escasa literatura referida a las multinacionales procedentes de América Latina. Los trabajos existentes se centran en el proceso de la multinacionalización de las empresas origen latinoamericano y en la evolución histórica que ha permitido su surgimiento y su configuración como referencias a nivel mundial en sus respectivos sectores. El trabajo analiza cómo dentro del espacio europeo, las multilatinas han validado su proceso de internacionalización con destino al espacio de la Europa Central; en concreto en los países del Grupo de Visegrád.
\end{abstract}

PALABRAS CLAVE: Multilatinas, CEMEX, Embraer, KATCON, NEMAK, Empresas de Mercados Emergentes, Grupo de Visegrád, internacionalización, Inversión Extranjera Directa

\section{ABSTRACT}

This article aims to expand the still limited literature referred to multinational enterprises from Latin America. Existing works focus on the historical evolution that has allowed the process of multinationalisation of Latin-American companies and their emergence and configuration as worldwide references in their respective sectors. This paper analyzes the applicability and validity of existing theoretical frameworks for the process of internationalization of multilatinas in the region of Central Europe; particularly in the countries of the Visegrád Group.

KEYWORDS: Multilatinas, CEMEX, Embraer, KATCON, NEMAK, Emerging Markets Multinationals, Visegrád Group, internationalization, Foreign Direct Investment

JEL: E22, F23, O52, O54

\section{INTRODUCCIÓN}

El objetivo de este trabajo es revisar las teorías principales de la internacionalización de las empresas y comprobar su validez con las observaciones empíricas obtenidas en la región del Grupo de Visegrád, compuesta por Eslovaquia, Hungría, Polonia y República Checa (en adelante V4). Los resultados obtenidos no pretenden sintetizar o generalizar las pautas del proceso de internacionalización que se han registrado en la región estudiada, sino que su propósito es confirmar o rechazar los enfoques 
Nedela, Daniel. Análisis de la presencia de las multilatinas en el espacio económico del grupo de Visegrád.

existentes respecto al fenómeno y verificar su adecuación al proceso de internacionalización de las empresas procedentes de América Latina.

La literatura económica que aborda la temática del proceso de transnacionalización de empresas procedentes de mercados emergentes es por ahora escasa. El retraso en abordar este fenómeno por la comunidad científica se debe a una solamente reciente emancipación de estas empresas con las multinacionales tradicionales del mundo occidental. La transnacionalización de las empresas emergentes no es un hecho nuevo, tiene sus orígenes en el siglo $\mathrm{XIX}^{1}$, lo que sí es novedoso es su impacto y las posiciones de liderazgo en sus respectivos sectores donde compiten en igualdad de condiciones con sus socios originarios de las economías desarrolladas.

Las "Multilatinas" es el término que se emplea para identificar a las empresas multinacionales procedentes de América Latina. Es una palabra relativamente nueva, ya que su empleo coincide con la fase de la expansión mundial de las empresas latinoamericanas que empieza a producirse a partir de la primera década del siglo XIX. Siendo un término reciente no está exento de polémica, ya que tanto el área de América Latina como el término multinacional carecen de significados precisos. (Á. Cuervo-Cazurra, 2010) Este trabajo utiliza la definición aplicada por la consultora Towers Watson, que por un lado, entiende como América Latina a todo el continente americano, incluidas las Islas del Caribe, pero sin contar EE.UU. y Canadá. Por otro lado, el término multinacional abarca a la totalidad de las empresas con operaciones de valor añadido en el exterior. Además, para que la empresa sea considerada como multilatina su sede principal debe estar localizada en América Latina y su actividad en prestación de servicios o producción de bienes debe llevarse a cabo en varios países que se encuentran por lo menos en dos continentes. (Towers Watson, 2010).

Las empresas nacidas en los países de América Latina han llegado a ser globales en un proceso de transnacionalización que Lourdes Casanova divide en tres etapas progresivas y dadas sus características bien diferenciadas. (L. Casanova, 2010) La primera fase se produce entre los años 1970 y 1990. Es en este periodo cuando ocurre la expansión inicial de las empresas nacionales de los países de América Latina a sus mercados limítrofes dentro de la misma región. La fase de expansión regional termina en la década de los noventa con la implantación del Consenso de Washington. La aplicación de la fórmula económica neoliberal marca el principio de la segunda fase del proceso descrito por Casanova y se caracteriza por dos elementos claves. En primer lugar, sucede la apertura de mercados a la competencia internacional. Tras este acontecimiento las empresas de propiedad pública, que hasta el momento disfrutaron de monopolios nacionales protegidos por el Estado, se encontraron con un entorno nuevo regido por las reglas del mercado. En segundo lugar, dado su bajo nivel de competitividad y escasa motivación por disminuir costes incurren en pérdidas y crisis financieras. Frente a la situación de una urgente necesidad de sanear los balances negativos se aprueban los procesos de privatización de las grandes empresas que anteriormente se denominaban campeones nacionales. Es sobre todo el caso de las empresas que operaron en

\footnotetext{
${ }^{1}$ Por ejemplo, la empresa de calzado argentina Alpargatas se convirtió en una multinacional en 1890 CuervoCazurra, Á. 2010. "Multilatinas." Universia business review, (25), 14-33.
} 
Nedela, Daniel. Análisis de la presencia de las multilatinas en el espacio económico del grupo de Visegrád.

sectores estratégicos como recursos naturales, telecomunicaciones, energía y finanzas. La tercera fase comienza con la expansión de la actividad internacional de estas empresas privatizadas a nivel global. Se trata de una fase que ha comenzado a partir del año 2002 y dura hasta el presente. Las empresas latinoamericanas que emprendieron este camino destacan por su ambicioso liderazgo que les convertirá posteriormente en líderes de sus sectores, configurándose de este modo como "multilatinas globales". Precisamente a lo largo de esta etapa las multilatinas llegan al espacio de la V4. La primera compañía latinoamericana en realizar este paso fue NEMAK, una empresa metalúrgica procedente de México, que tras materializar una inversión greenfield en la República Checa en el año 2004, empieza a explorar un espacio hasta entonces desconocido. Esta empresa hizo de punta de lanza para que posteriormente la presencia de las multilatinas aumentase en V4 con el establecimiento de otras tres empresas industriales: CEMEX (en 2005), KATCON (en 2009) y Embraer (en 2011). Para completar el escenario de las multilatinas en la región hay que mencionar también a la consultora brasileña Stefanini IT Solutions. Siendo ésta una empresa dedicada a la industria del conocimiento, su proceso de transnacionalización no exige de un esfuerzo elevado en comparación con las cuatro empresas anteriormente mencionadas. A lo largo del trabajo solamente se mencionarán algunas características de su presencia, manteniendo el enfoque principal en las empresas dedicadas a la industria tradicional.

Para los propósitos mencionados, en primer lugar se revisarán las principales teorías que se preocupan por el fenómeno de la internacionalización de las empresas, aplicando este marco teórico a las multilatinas presentes en la V4 y en tercer lugar evaluar si dicho marco es adecuado para explicar su llegada a dicha región. El análisis que se llevará a cabo consiste en un estudio detallado de las cinco empresas latinoamericanas mencionadas anteriormente. Las aportaciones que se realizarán emanan de la premisa presentada en la hipótesis elaborada por Lourdes Casanova para una publicación del Banco Interamericano de Desarrollo (L. Casanova et al., 2009) donde se defiende la idea de las multilatinas como jugadores globales. Ya de antemano podemos confirmar esta hipótesis con el simple hecho de que las empresas que son objeto de esta investigación están activamente presentes en una región como es la $\mathrm{V} 4$, es decir, en un espacio muy poco conocido para ellas, con unas escasas relaciones bilaterales de inversión y comercio que de manera agrupada acumulan cifras en torno a $1 \%$ del total de los flujos individuales entre las dos regiones.

\section{MARCO TEÓRICO}

Los estudios sobre la complejidad de los procesos de transnacionalización de las empresas multinacionales surgieron a partir de los años setenta y progresivamente se fueron ubicando en varios campos propios de la ciencia económica y de las ciencias empresariales. Los enfoques de estudios de los que disponemos hoy en día centran su atención en una variedad de aspectos. Para el presente trabajo se han elegido los paradigmas capaces de reflexionar sobre las decisiones gradualistas de los empresarios, sobre la configuración del poder de las empresas, sobre las ventajas proporcionadas por la misma transnacionalización y sobre la misma esencia de la transnacionalización basada en la Inversión Extranjera Directa (IED). Las teorías que recogen los conceptos mencionados se presentarán en el subapartado 1.1. 
Nedela, Daniel. Análisis de la presencia de las multilatinas en el espacio económico del grupo de Visegrád.

Una vez analizadas las principales escuelas generales de la transnacionalización se procederá a la descripción del marco conceptual específico, extraído de la literatura referida a las multilatinas en particular. Entre estos estudios destacan los trabajos que correlacionan la IED con el nivel del desarrollo y con las características económico-institucionales de los países latinoamericanos y los que analizan la dirección geográfica de la primera expansión internacional de las empresas latinoamericanas. El recorrido a través de las aportaciones mencionadas tendrá lugar en el subapartado 1.2.

\subsection{Teorías tradicionales de la internacionalización de las empresas}

Los economistas J. Johanson y J. E. Vahlne han sido unos de los primeros autores en abordar el fenómeno de la internacionalización de las empresas, configurando así la Escuela de Uppsala. (J. Johanson y J. E. Vahlne, 1977) En sus planteamientos, las empresas de manera gradual aumentan su actividad internacional, por lo que la internacionalización es vista como el producto de una serie de decisiones incrementalistas. Para emprender este camino incrementalista la variable "conocimiento" es considerada como crítica, debido a que es necesaria para la expansión internacional de la empresa, pero a su vez, solamente se puede adquirir con el mismo proceso de internacionalización. Los estudios de la Escuela de Uppsala se fundamentan en una evidencia empírica que permite observar que la internacionalización de una empresa se produce primero vía exportaciones. En el caso de que los beneficios del comercio exterior evolucionaran positivamente se puede establecer un mayor compromiso por medio de la instalación de agentes y filiales de venta. A mayor éxito de éstos aumenta tanto la cuota de mercado de la empresa, como el compromiso con el mercado, lo que en la última instancia culmina con la instauración de las líneas propias de producción en el territorio foráneo. La selección del destino para emprender la senda expansiva de la actividad internacional también forma parte del objeto de estudio de la Escuela de Uppsala y según sus planteamientos obedece dos variables. En primer lugar, figura la distancia física y la facilidad de acceso al mercado en cuestión. En segundo lugar, está el papel que desempeñan las diferencias culturales que engloban al idioma, educación, prácticas empresariales, y los modos de vida en general.

Identificando el destino para la expansión de la actividad económica de las empresas tenemos que preguntar también “¿el por qué?' de la transnacionalización. Dentro del marco de la Escuela de Uppsala, la entrada a los mercados foráneos se produce como una adaptación a las condiciones cambiantes en el entorno de la empresa. La expansión internacional de la empresa es una respuesta a nuevos problemas y oportunidades que se ve fomentada por la disponibilidad de conocimiento de mercado. El conocimiento es entendido como la experiencia adquirida con el mercado y a medida que aumenta, la empresa está más capacitada para identificar con una mayor fiabilidad el riesgo existente. En el caso de que el riesgo existente sea inferior al riesgo máximo tolerable, las decisiones de la empresa toman el sentido incrementalista apuntando por Johanson y Vahlne con el deseo de adquirir un compromiso mayor con el mercado y de este modo aprovechar la oportunidad presente.

Hoy en día, el marco analítico más extendido entre la comunidad científica sobre la transnacionalización de las empresas es el paradigma ecléctico, también llamado 
Nedela, Daniel. Análisis de la presencia de las multilatinas en el espacio económico del grupo de Visegrád.

"modelo OLl", elaborado por John H. Dunning (J. H. Dunning, 1999). El paradigma OLI no constituye una teoría formal pero sí permite la categorización, análisis y estudio empírico de la IED. OLI, en sus siglas en inglés significa Ownership, Location and internalization (propiedad, localización e internalización). Cada uno de estos elementos proporciona motivos específicos que conducen hacia la internacionalización de la actividad económica de las empresas independientemente de su origen. Entre las ventajas relacionadas con la propiedad, las multilatinas están buscando nuevos mercados y el aumento de su tamaño. Respecto a la localización, los atributos que se persiguen están vinculados con la eficiencia, como el aumento de tamaño, pero también aparecen otros elementos relacionados con la búsqueda de factores de producción más baratos o más cualificados, o el acceso a mercados de capital donde el coste de la financiación es más reducido. Dado que los costes laborales son más elevados en Europa que en América Latina, en nuestro caso no se está persiguiendo una eficiencia vía precio sino vía calidad, mediante el acceso a tecnologías y medios de producción que mejoran los resultados obtenidos en términos de calidad fabril, durabilidad e imagen. A esta estrategia se adecua también el modo de entrada de las multilatinas al mercado europeo. La herramienta más empleada por las multilatinas son las fusiones y adquisiciones con empresas que ostentan una elevada cuota de mercado y cuentan con una plantilla especializada y con experiencia en su sector de actividad. (Towers Watson, 2013) El tercer elemento relacionado con las ventajas propias de la internalización está configurado por las virtudes aportadas por los dos elementos previos.

Un tercer modelo capaz de explicar la transnacionalización de las empresas multinacionales es el modelo de Alan M. Rugman que centra su atención en los fundamentos microeconómicos de la competitividad de las empresas. El modelo de Rugman está basado en la identificación de las ventajas específicas, por un lado, de la empresa (FSA - Firm Specific Advantages) y por el otro, del país de origen de la misma (CSA - home Country Specific Advantages) (A. M. Rugman, 2010) De acuerdo a esta teoría, en principio las empresas procedentes de países emergentes carecen de las ventajas específicas propias y deben aprovechar las proporcionadas por su país de procedencia. En América Latina la configuración habitual de los mercados nacionales consistía en ofrecer a las empresas públicas el poder monopolístico sobre la producción creando economías de escala. De este modo el marco legal de los Estados latinoamericanos proporcionó las CSA, y al mismo, configuró las FSA iniciales de los campeones nacionales. Debido a que las FSA configuradas estaban basadas exclusivamente en una cuota de mercado monopolística, las empresas no se encontraban en una posición adecuada para competir con las multinacionales de los países desarrollados en los mercados internacionales. Coincidiendo con las observaciones empíricas de L. Casanova (L. Casanova, 2011a) y con el marco teórico de la Escuela de Uppsala, el inicio de la transnacionalización de los campeones nacionales ocurre en su región natal minimizando de este modo el riesgo y aprovechando las similitudes culturales. La primera expansión internacional de las empresas monopolísticas es clave desde el punto de vista de la competencia ya que están obligadas a acomodar sus productos de acuerdo a las demandas del consumidor y disminuir los costes para ganar cuota en un mercado donde la legislación de su Estado protector no es aplicable. La expansión regional de las empresas públicas de América Latina ha producido una integración económica de facto por medio de una interdependencia mutua e influencia recíproca de los países latinoamericanos y a su vez ha fortalecido las FSA 
Nedela, Daniel. Análisis de la presencia de las multilatinas en el espacio económico del grupo de Visegrád.

de dichas empresas. ${ }^{2}$ Dado que el robustecimiento de las FSA se sustenta en el conocimiento y en el poder económico de la empresa, la mejora de las FSA constituye el punto de partida para una progresiva internacionalización. Estos dos elementos, en fases más avanzadas, permiten el uso de las tácticas de lobby que posteriormente posibilitan la creación de las relaciones clientelares entre empresarios y gobiernos, ofreciendo unas posibilidades mayores de crecimiento de la empresa.

A causa de un desarrollo económico tardío de los mercados emergentes y de una solamente reciente emancipación de sus empresas con las procedentes de los países desarrollados, según Rugman, las multilatinas son unos "late comers" al escenario de la economía mundial. Su posición de partida está caracterizada por un entorno mucho más complejo que el experimentado por sus competidores de países desarrollados que se transnacionalizaron previamente. Primero, las Compañías Transnacionales (CTN) emergentes utilizaron sus FSA para las operaciones intrarregionales donde adquirieron los conocimientos y recursos necesarios para una expansión mundial que posteriormente les permitió convertirse en jugadores globales. Los motivos que las llevaron a este camino son la necesidad de adquirir conocimientos y experiencias de management por lo que muchas veces su estrategia de modo de entrada a los mercados foráneos se basa en fusiones y adquisiciones de otras empresas que incluyen el traspaso tanto de tecnología, como de conocimientos y del personal directivo. Un estudio publicado por Towers Watson en 2013 demuestra que la obtención y la retención del personal directivo con experiencia es el reto más acuciante de las empresas emergentes tras una operación de fusión o adquisición. Las multilatinas que utilizaron esta herramienta para entrar en un mercado extranjero emplearon una filosofía basada en la conquista repentina del nuevo mercado gracias a una compra del know-how intrínseco de la empresa contenido en la misma operación de compra de activos. (Towers Watson 2013)

Por último, respecto a las teorías generales de la transnacionalización de la actividad empresarial, recurrimos a la tesis denominada "¿Porque el capital no fluye de los países ricos a los pobres?", en la que R. Lucas aplica la hipótesis del rendimiento decreciente a los flujos de capitales entre los países ( $R$. E. Lucas, 1990). En teoría, el capital debería fluir de los países más ricos hacia los más pobres para aprovecharse del rendimiento original, pero Lucas descubre que este pretexto no se cumple. Durante la década de los noventa la mayoría del flujo de la inversión extranjera directa se efectuaba entre los países ricos y dentro del mundo desarrollado. En su análisis identifica cuatro razones que causan que el rendimiento marginal se iguale o que hasta beneficie a los países donde debería ser más bajo. Aplicando el modelo de Lucas observamos, en primer lugar, que las diferencias en capital humano causadas por la cualificación de la mano de obra a raíz del nivel educativo, estructura de edad o de sector en cuestión benefician a los países desarrollados. En segundo lugar, el capital humano también presenta beneficios externos en sí mismo por proporcionar una mayor dotación tecnológica reflejada en

\footnotetext{
${ }^{2}$ Integración económica de facto - A causa de una intensificación de las relaciones empresariales entre los países de una región determinada, las economías que la componen se convierten progresivamente en espacios económicos interdependientes y conjuntamente integrados en la economía mundial. El proceso de una integración de facto ocurre sin la necesidad de una voluntad política que expresara explícitamente tal deseo por medio de un acuerdo bilateral vinculante.
} 
Nedela, Daniel. Análisis de la presencia de las multilatinas en el espacio económico del grupo de Visegrád.

la estructura de la producción de la economía haciendo el país desarrollado más atractivo para la inversión, tanto por contar con una mano de obra cualificada como por poder esperar un "spillover" tecnológico en beneficio de la empresa. En tercer lugar, las imperfecciones del mercado de capitales causadas por el comportamiento monopolístico u oligopolístico de las empresas multinacionales producen distorsiones en los flujos de la inversión extranjera directa. Y en el último lugar, nos encontramos con el factor denominado "riesgo político" que está relacionado con la solidez y solvencia institucional. Este cuarto elemento influye de manera directa sobre los flujos de la IED y en gran medida también sobre el nivel de desarrollo del país. Considerando las variables como el riesgo de expropiación o los problemas relacionados con altos niveles de corrupción, entre otras variables institucionales, los países ricos presentan un entorno empresarial más propicio para la inversión. El rendimiento marginal decreciente se equipara entonces gracias a los atributos propios de su espacio económico, ofreciendo mayores rentabilidades y garantizando la permanencia de la inversión independientemente del escenario político. Utilizando los esquemas de Lucas podemos concluir que una vez que las multilatinas se sienten fortalecidas suficientemente, dirigen su inversión extranjera a los países con un nivel de desarrollo superior al de su propio país. Este proceso corresponde a la tercera fase de la expansión internacional de las multilatinas. Es decir, al momento cuando éstas, motivadas por la búsqueda de la eficiencia vía calidad se ven obligadas a invertir en países con un tejido tecnológico más desarrollado y con una estabilidad institucional mayor.

\subsection{La internacionalización de las multilatinas}

Tras haber realizado un recorrido por la literatura general dedicada a la transnacionalización de las empresas, procedemos analizar las principales aportaciones que se refieren directamente al fenómeno de las multilatinas; entre estas destacan las contribuciones de los autores como Alonso (F. Alonso y J. Revuelta, 2013), Casanova (L. Casanova, 2010, 2011b, L. Casanova et al. 2009, L. Casanova y E. Rodríguez-Montemayor, 2014), Cuervo-Cazurra (Á. Cuervo-Cazurra, 2008, 2010), Fleury (A. Fleury, 2010, A. Fleury et al., 2013), Rivera (R. Rivera y A. Ruelas-Gossi, 2007) o Santiso (E. Frot y J. Santiso, 2013, J. Santiso, 2011, 2013).

Empezando por la dimensión geográfica destaca la teoría "The bridge modep", elaborada por Casanova y Rodríguez-Montemayor, que a base de evidencia empírica revela que España y Portugal funcionan como un "hub latino", es decir, que las multilatinas presentes en la UE deciden realizar su primera expansión hacia los dos países mencionados. (L. Casanova y E. Rodríguez-Montemayor, 2014) De acuerdo a su hipótesis, encuentran razones que motivan este primer paso en los objetivos perseguidos por las empresas en cuestión e identifica cinco tipos de puentes que permiten dicha expansión: puente comercial, puente de negocios, puente de conocimientos, puente financiero y puente logístico. Dada la cercanía relativa de América Latina y Península lbérica, el buen estado de las infraestructuras, experiencia ibérica en Europa continental y la historia compartida y reforzada por los lazos coloniales, las multilatinas consideran a España y Portugal como los destinos más propicios para acceder a una financiación más barata y para acercarse a los potentes mercados europeos. Por un lado, España cumple esta función para las empresas procedentes de los países hispanohablantes, mientras que Portugal la cumple para el caso de Brasil. La hipótesis de España como un "hub 
Nedela, Daniel. Análisis de la presencia de las multilatinas en el espacio económico del grupo de Visegrád.

latino" es soportada también por los trabajos empíricos de Santiso. De acuerdo a sus observaciones, aproximadamente un $70 \%$ de las empresas con origen latinoamericano establecen sus sedes europeas precisamente en España, entre estas empresas figuran CEMEX, PEMEX, Alpargatas y Arcor entre otras. (J. Santiso, 2013)

En lo referido a la IED saliente de América Latina, Stal y Cuervo-Cazurra clasifican los países en base a su nivel de desarrollo que según la teoría está positivamente correlacionado con el flujo de la IED. (Á. Cuervo-Cazurra y E. Stal , 2011) La clasificación establece cinco niveles progresivos de desarrollo con los que se corresponden unas características determinadas de la IED, empezando con el nivel de los Países Menos Adelantados con un flujo mínimo de IED en ambos sentidos, terminando con los países desarrollados con altos niveles de IED entrante y saliente. Los países de América Latina se encuentran en el segundo o en el tercer eslabón del flujo de la IED. Por un lado, los países latinoamericanos con empresas internacionales propias se sitúan en el tercer eslabón, es decir, que son receptores netos, pero ya registran ciertos niveles de la IED saliente. Las empresas transnacionales de estos países concentran su actividad en un campo específico y por medio de inversiones extranjeras intentan convertirse en líderes sectoriales. Este primer grupo de países está encabezado por Brasil y México seguidos por Chile, Argentina, Colombia y Venezuela. Por otro lado, el segundo grupo está formado por países que solamente reciben la IED sin que existieran flujos relevantes de salida de capitales. En general estos países suelen disponer de empresas pequeñas, que junto con la entrada de la IED, se ven amenazadas por los jugadores internacionales y motivadas por el miedo a la competencia adquieren experiencia necesaria para una posterior expansión internacional dentro del ámbito regional. Por último, existe un tercer grupo de países que se escapa a la clasificación básica, es el caso de los paraísos fiscales que funcionan como centrifugadores de capitales. Los países catalogados como territorios de baja tributación presentan los niveles de IED emitida muy por encima de su nivel de desarrollo. Se trata de una anomalía causada por una legislación muy laxa respecto al control de capitales.

Cuervo-Cazurra profundiza en su clasificación y encuentra una explicación del surgimiento de la IED saliente también más allá del nivel de desarrollo. Esta segunda clasificación agrupa los emisores de la IED según las variables población y apertura comercial. (Á. Cuervo-Cazurra, 2010) En este caso nos encontramos con tres grupos de inversores. En primer lugar, se encuentran los países con mucha población y un fuerte mercado interior que permite a los antiguos monopolios estatales tener un tamaño suficiente para competir con las empresas extranjeras e internacionalizar su actividad. Entre estos países figuran Brasil, México o Argentina. En segundo lugar, está el grupo de países con población pequeña y un mercado con altos niveles de apertura internacional. En este caso una alta exposición a la economía mundial ha causado mejoras en la competitividad de las empresas nacionales que, al mismo tiempo, se aprovecharon de las pocas barreras existentes para invertir en el exterior. Uno de estos países es Chile. El último grupo está conformado por la anomalía de paraísos fiscales, ya mencionados anteriormente. Es el caso de países con población y mercado pequeño, pero con unas tasas 
Nedela, Daniel. Análisis de la presencia de las multilatinas en el espacio económico del grupo de Visegrád.

impositivas ínfimas como por ejemplo las Islas Caimán o Panamá, y en otra medida Uruguay. ${ }^{3}$

Para Cuervo-Cazurra, además de estas clasificaciones, también existen ciertos factores "push" que estimulan las decisiones empresariales de expansión internacional, fomentando de este modo la salida de la IED. Entre estos factores figura el entorno macroeconómico e institucional. Una vez aplicadas estas variables a los principales emisores de la IED de América Latina descubrimos un escenario en el que las crisis económicas ocurren con alta frecuencia, existen altos índices de corrupción y en algunos casos también está presente la inestabilidad política. Estos factores pueden fomentar una salida previa de capitales a la correspondiente a su nivel de desarrollo. Tampoco hay que olvidarse del hecho de que además de las razones institucionales que motivan la internacionalización se hallan también razones puramente microeconómicas, como la búsqueda de ventaja competitiva o acceso a tecnología y factores de producción sofisticados.

\section{PRESENTACIÓN DE LOS CASOS EN EL CONTEXTO DE LA V4}

La última década ha sido testigo de la expansión mundial de las empresas procedentes de los mercados emergentes y las multilatinas han seguido este rumbo hasta el punto en que, hoy en día, marcan las tendencias en algunos sectores. Los países emergentes y sus empresas han aprovechado el contexto de crisis económica que desde el año 2007 sacude al mundo occidental y bajo este prisma en el que las empresas multinacionales tradicionales se encuentran con una necesidad urgente de financiación, los Estados de la Unión Europea están inmersos en la crisis de su deuda externa y EE.UU. registra una recuperación muy lenta. Mientras, el entorno macroeconómico de los países emergentes es propicio para el fomento de la transancionalización de sus empresas ansiosas de conquistar cuotas de mercado más allá de las fronteras nacionales. No solo es resultado de las elevadas tasas de crecimiento interanual del PIB que estos países registran, sino que tanto el poder adquisitivo de su población como los beneficios repatriados por las empresas, que ya se han multinacionalizado, generan un fuerte empuje para la expansión a los mercados extranjeros.

En este apartado la expansión de las multilatinas a Europa Central, en concreto al espacio del Grupo de Visegrád es el punto de reflexión. La hipótesis se sustenta en la idea de la existencia de penetración cada vez mayor de las economías emergentes en las desarrolladas como proponía Lucas. Es decir, que la tendencia tradicional de una empresa occidental que internacionaliza su actividad hacia los diferentes mercados emergentes ya no es válida para todos los procesos de expansión internacional de la actividad económica privada. Desde la última década del siglo XX estamos registrando el flujo de la IED en dirección inversa que con el paso de los años se intensifica y que desde el momento de la irrupción de la crisis de las economías occidentales en 2007 lejos de desaparecer, empieza cobrar más importancia. El escenario actual difiere del observado antes de la crisis, tanto por la aparición de empresas procedentes de mercados emergentes como por la conquista

\footnotetext{
${ }^{3}$ Los países incluidos en la lista de los paraísos fiscal de la UE están excluidos de este trabajo debido a una procedencia inexacta de la inversión. http://ec.europa.eu/taxation_customs/taxation/gen_info/good_governance_ matters/lists_of_countries/index_en.htm
} 
Nedela, Daniel. Análisis de la presencia de las multilatinas en el espacio económico del grupo de Visegrád.

de posiciones de liderazgo en sus sectores de actividad. En este contexto cambiante América Latina no se ha quedado atrás y el flujo de su IED ha crecido desde el año 2000 de manera significativa aunque presentando unas fluctuaciones significantes. Según los datos de la UNCTAD, la IED saliente del Grupo Latinoamericano ha pasado de representar una cuota mundial en torno a $0,677 \%$ del total de la IED mundial en el año 2000 a una cuota de 2,258\% en el año 2013. Durante este periodo el año más sobresaliente para la IED latinoamericana fue 2012 con una cuota del 3,307\% del total del flujo saliente de IED. Parte de estas inversiones ha sido destinada también a la región V4.

\subsection{Embraer - milagro brasileño en tecnología de punta}

Empezando por el caso más emblemático, nos encontramos con la presencia de la multinacional brasileña Embraer (Empresa Brasileira do Aeronáutica) en la República Checa. La compañía Embraer es un ejemplo de empresa que con su posición en el mercado rompe los paradigmas desarrollistas y estructuralistas elaborados por los pensadores económicos de la economía del desarrollo para salir del círculo vicioso de la pobreza descrito por R. Nurkse (R. Nurkse, 1952) y por no coincidir con la tradicional división internacional del trabajo descrita por C. Furtado y R. Prebisch (C. Furtado, 1974, R. Prebisch, 1964). Según estas escuelas, sería imposible que una empresa de tecnología de punta como Embraer pudiera surgir fuera de lo que Amín denominó como "centro", es decir en la "periferia". (S. Amin, 1988) A pesar del pronóstico desfavorable la empresa brasileña Embraer demuestra que es capaz de competir a nivel mundial en su sector de actividad y además se ha convertido en líder mundial en la producción aeronáutica entre los fabricantes de aviones con capacidad entre 70 y 120 personas.

¿Pero cuál ha sido la estrategia que permitió a Embraer convertirse en uno de los jugadores principales en su sector a nivel mundial y porqué estableció una Joint Venture con AERO Vodochody (República Checa) y de este modo penetró en el espacio del V4? La Empresa Brasileira do Aviación se fundó en el año 1969 como una empresa pública durante el periodo de sustitución de importaciones a raíz de la propuesta realizada por el Ministerio de Defensa de Brasil, que en su momento consideró de importancia crucial desarrollar una línea de fabricación de aeronaves propia. Una vez terminado el periodo de sustitución de importaciones y empezada su privatización en los años noventa, la empresa se reestructuró y progresivamente se convirtió en el tercer fabricante de aviones en su categoría a nivel mundial. Solamente por detrás de Boeing y Airbus y delante de otras compañías que han surgido en mercados desarrollados y en unas condiciones más favorables a las de Embraer, como la canadiense Bombardier Aerospace, estadounidense Lockheed Martin o sueco SAAB (A. Goldstein, 1995).

La colaboración entre Brasil y República Checa en terreno aeronáutico comienza en el año 2009 cuando tuvo lugar una visita oficial del Ministro de Defensa de la República Checa a Brasil, seguida por la celebración de una feria de tecnología checa organizada por la agencia promotora de inversiones Czechinvest, cuyo propósito era fomentar la cooperación bilateral en el campo de la industria aeronáutica. Los dos eventos culminaron con una declaración de intenciones común entre los Ministerios de Defensa de ambos países con fecha de septiembre de 2010. Posteriormente, en abril de 2011, durante la Feria de Tecnología de Defensa 
Nedela, Daniel. Análisis de la presencia de las multilatinas en el espacio económico del grupo de Visegrád.

(LAAD), organizada por el gobierno brasileño, Embraer y AERO Vodochody firman un acuerdo de colaboración a través de una Joint Venture para el desarrollo y fabricación del nuevo modelo de avión denominado KC-390. ${ }^{4}$

Aero Vodochody es una empresa de aviación checa fundada en el año 1919, que al igual que su socio brasileño ha pasado por un periodo de una crisis grave en los años 90. La empresa Boeing adquirió Aero Vodochody en el año 1998 y bajo la bandera del gigante estadounidense la compañía ha sido capaz de mantener su propia producción. En el año 2004 Aero Vodochody fue comprada por su propietario actual, un grupo inversor eslovaco, Penta Investments. Desde entonces Aero Vodochody consigue establecer Joint Ventures con los principales fabricantes de aviones y helicópteros como Airbus, Sikorsky, Bombardier Aerospace y también, para lo que nos concierne, con Embraer.

El éxito del fabricante checo es una consecuencia directa del apoyo del Banco de Exportaciones de la República Checa, cuyo objetivo es mejorar la competitividad de la economía del país por medio de subvenciones a las exportaciones y fomentar su participación en la economía global a través de la industrialización orientada hacia el exterior. Independientemente del apoyo público, también cabe mencionar una larga trayectoria y buena reputación del fabricante en el campo de la aviación.

El desarrollo del proyecto de KC-390 surge a raíz de una iniciativa emprendida por el Ministerio de Defensa de Brasil bajo el plan de modernización de la flota aeronáutica del país. Sus orígenes se remontan al año 2008 cuando el Congreso Nacional del Brasil aprueba una subvención de 440 millones de dólares para el desarrollo del proyecto de diseño y fabricación del modelo KC-390. ${ }^{5}$ En 2009 Embraer gana el contrato para desarrollar y construir dos prototipos del avión mencionado por un valor aproximado de 1.500 millones de dólares. ${ }^{6}$

El avión KC-390 es una aeronave multiusos de tamaño medio especializada en el transporte de bienes y personas que se puede utilizar tanto en operaciones civiles como militares. El desarrollo y la fabricación del avión es el resultado de una colaboración internacional entre los fabricantes aeronáuticos de cuatro países: Brasil, Argentina, Portugal y República Checa. Posteriormente al proceso de fabricación y ensamblaje del avión se unieron también dos empresas aeronáuticas de Chile y Colombia. El proyecto vio la luz en octubre de 2014 cuando el nuevo avión KC-390 fue presentado por primera vez y posteriormente en enero de 2015 realizó con éxito el primer vuelo de prueba. Durante la presentación del avión los representantes de Embraer han mencionado en reiteradas ocasiones a la República Checa como socio importante y fiable para una futura colaboración. ${ }^{7}$

\footnotetext{
4 http://www.czechinvest.org/svetovy-vyrobce-letadel-embraer-nasel-partnera-v-ceske-republice Publicado: 27/04/2011 Consultado: 19/10/2015

5 http://www.blogdorogerio.com.br/2007/11/19/camara-federal-aprova-orcamento-de-r-800-milhoes-para-aviaocargueiro-militar/ Publicado: 19/11/2007 Consultado: 19/10/2015

6 http://brasil.estadao.com.br/noticias/geral,defesa-salva-de-cortes-plano-de-aviao-cargueiro-imp-,761590 Publicado: 21/10/2011 Consultado: 19/10/2015

${ }^{7} \mathrm{http}: / /$ www.prumysl.cz/brazilsky-vojensky-letoun-embraer-poprve-vzletl/ Publicado: 05/02/2015 Consultado: $19 / 10 / 2015$
} 
Nedela, Daniel. Análisis de la presencia de las multilatinas en el espacio económico del grupo de Visegrád.

A fecha de hoy ya se han acordado los primeros contratos de compra sobre este modelo de avión, tanto por el sector militar como por el civil. Los ministerios de defensa de varios países ya han firmado los contratos vinculantes para la compra de KC-390. La lista está encabezada por Brasil con un pedido de 28 aeronaves, seguido por Colombia con 12, Portugal y Chile con 6 y la República Checa con 2 unidades. ${ }^{8}$ También está en proceso de negociación la firma de contratos con Francia, Suecia y Perú. El resultado de las negociaciones con los países de Europa Occidental será clave para confirmar la hipótesis de una elevada competitividad y calidad de productos de las empresas emergentes en general y en particular de las multilatinas, debido a que tanto Suecia como Francia disponen de sus propias líneas de fabricación de aeronaves capaces de desarrollar y producir un avión similar al modelo KC-390. Independiente del interés de los países de Europa Occidental en adquirir los aviones del fabricante brasileño, según el director ejecutivo de Embraer, Frederico Fleury Curado, los principales mercados para el KC-390 se encontrarán en América Latina, Asia y África por su bajo coste comparativo respecto a modelos equivalentes elaborados por la competencia. ${ }^{9}$ Para facilitar la venta y la comercialización, Embraer ha firmado un Acuerdo de Entendimiento con Boeing que se ha comprometido como el interlocutor para los contratos que se establecerán en EE.UU., Reino Unido y Oriente Medio. ${ }^{10}$ Por el lado civil, La Empresa Brasileña de Correos ${ }^{11}$ ha mostrado interés en adquirir aeronaves, pero el contrato todavía sigue en negociación. Todos los contratos mencionados, tanto los concluidos como los que están en fase de negociación, se basan en la estrategia de bajo precio y calidad. El precio estimado de Embraer KC-390 será aproximadamente unos 50 millones de dólares, un $20 \%$ menos en comparación con las aeronaves de características similares de otros fabricantes.

El establecimiento de la Joint Venture entre Embraer y Aero Vodochody implica la participación de la empresa checa en la fabricación de la cola del avión, de la rampa de carga y de las puertas de la cabina, así como en el desarrollo y construcción de las alas. De acuerdo a la teoría de Casanova en el desarrollo de las alas del avión se daría un caso de innovación a la inversa.(L. Casanova, 2010) El fenómeno que se da cuando una empresa se multinacionaliza por medio de adquisiciones o Joint Ventures hacia los países de un nivel tecnológicamente más elevado para conseguir dicha tecnología y aplicarla en sus propios procesos de producción.

Según las declaraciones del presidente de Aero Vodochody, el valor estimado de la producción de las partes encargadas alcanzará, durante los primeros seis años de producción, fruto de la colaboración, unos 222,8 millones de dólares y a su vez se espera una progresiva ampliación de la producción hasta fabricar unos 700 aviones para el año 2030. Aero Vodochody se ha beneficiado de la colaboración con los fabricantes extranjeros, entre ellos Embraer, y gracias a estos contratos y Joint

\footnotetext{
8 https://www.flightglobal.com/news/articles/laad-embraer-expects-first-operational-kc-390-buyer-in-384442/ Publicado: 10/04/2013 Consultado: 19/10/2015

9 http://www.reuters.com/article/2013/09/19/us-embraer-kc390-insight-idUSBRE98I0BW20130919 Publicado: 19/09/2013 Consultado: 19/10/2015

10 http://www.embraer.com/en-us/imprensaeventos/press-releases/noticias/pages/boeing-e-embraer-assinamacordo-de-cooperacao-para-o-programa-kc390.aspx Publicado: 26/06/2012 Consultado: 19/10/2015

11 Empresa Brasileira de Correios e Telégrafos (ECT) o simplemente, Correios, es una empresa pública responsable de prestar el Servicio Postal Universal en todo el territorio de Brasil. La empresa es obligada por la ley prestar el servicio postal en todo el territorio del país, incluidas las zonas de difícil acceso terrestre.
} 
Nedela, Daniel. Análisis de la presencia de las multilatinas en el espacio económico del grupo de Visegrád.

Ventures ha conseguido a lo largo del año 2013 incrementar sus ventas en un 29\%, hasta los 154,1 millones de dólares, con lo que ocupa el número 100 del ranking mundial de las empresas aeroespaciales según el volumen de ventas. (Price Waterhouse Coopers (PwC), 2014) En 2013, la empresa proporcionaba unos 1.300 empleos directos de los cuales aproximadamente 250 se atribuyen a la colaboración con Embraer. ${ }^{12}$

El futuro del proyecto KC-390 apoyado por el Ministerio de Defensa de Brasil empieza a encontrarse con problemas de financiación debido a que el gobierno brasileño, vía el Ministerio de Defensa, anunció unos posibles recortes en el presupuesto de defensa que podrían afectar la financiación del desarrollo del avión, terminando de este modo su participación en el proyecto. Las declaraciones del director ejecutivo de Embraer describen la situación en la que se encuentra el proyecto y su relación con el Ministerio de Defensa afirmando que, a lo largo del año 2014 no se ha cumplido el flujo de 404 millones de dólares a cobrar del ministerio por parte de Embraer, y según su propia previsión el año 2015, será igualmente deficitario, esta vez en una cuantía inferior, en aproximadamente 100 millones de dólares. Los representantes de Embraer desaconsejan a las autoridades la suspensión de los fondos debido a que el proyecto del avión ya está desarrollado y que además ya existe una unidad capaz de volar. En el caso de que gobierno de Brasil abandonara el proyecto, Embraer está preparado para buscar recursos adicionales de financiación con otros países que colaboran en el desarrollo del proyecto de KC-390. ${ }^{13}$

El resultado del establecimiento de la Joint Venture entre Embraer y AERO Vodochody ha afectado la estructura de comercio bilateral entre la República Checa y Brasil. Desde la celebración del acuerdo, la partida número 88 del comercio exterior que agrupa las aeronaves, los vehículos espaciales y sus partes ha pasado de representar una cantidad ínfima a convertirse en una de las partidas más importantes del flujo comercial entre los países mencionados. El aumento del valor absoluto de las importaciones de Brasil agrupadas bajo la partida 88 ha aumentado entre el año 2008 y 2014 por 114 veces y las exportaciones en el mismo periodo por más de mil veces convirtiéndose en el primer rubro por valor absoluto en lo que se refiere a las exportaciones de Brasil hacia República Checa.

Valor absoluto y puesto relativo de la partida 88 (aeronaves, vehículos espaciales y sus partes) en el comercio bilateral entre República Checa y Brasil (visto desde Brasil)

\begin{tabular}{|l|c|c|c|c|c|c|c|}
\hline & $\mathbf{2 0 0 8}$ & $\mathbf{2 0 0 9}$ & $\mathbf{2 0 1 0}$ & $\mathbf{2 0 1 1}$ & $\mathbf{2 0 1 2}$ & $\mathbf{2 0 1 3}$ & $\mathbf{2 0 1 4}$ \\
\hline $\begin{array}{l}\text { Importación } \\
\text { (puesto) }\end{array}$ & 429 & 411 & 14199 & 34358 & 35924 & 28426 & 49121 \\
$(26)$ & $(25)$ & $(8)$ & $(4)$ & $(5)$ & $(6)$ & $(4)$ \\
\hline $\begin{array}{l}\text { Exportación } \\
\text { (puesto) }\end{array}$ & 24 & 25 & 13 & 352 & 9391 & 26008 & 27011 \\
& $(50)$ & $(43)$ & $(43)$ & $(22)$ & $(2)$ & $(1)$ & $(1)$ \\
\hline
\end{tabular}

Fuente: International Trade Center (ITC)

\footnotetext{
12 http://www.prumysl.cz/trzby-aera-z-vyroby-casti-embraeru-prekroci-pet-miliard/ Publicado: 05/11/2014 Consultado: 19/10/2015

13 http://zpravy.e15.cz/byznys/prumysl-a-energetika/brazilsky-embraer-trapi-finance-cesi-zatim-dodavaji-podleplanu-1179649 Publicado: 12/04/2015 Consultado: 19/10/2015
} 
Nedela, Daniel. Análisis de la presencia de las multilatinas en el espacio económico del grupo de Visegrád.

\subsection{CEMEX - líder global en la industria de materiales para la construcción}

CEMEX es una compañía mexicana fundada en el año 1906 especializada en cemento y hormigón. A lo largo de su historia sus hitos más remarcables tuvieron lugar en los años 1976 cuando la compañía se convierte en el productor de cemento más importante de México, 1985 cuando con la firma del GATT empieza a emprender su actividad internacional vía exportaciones y en 1992 emprende su expansión internacional con la adquisición de Valenciana de Cementos y Sansón, las dos plantas cementeras más grandes de España. (D. R. Lessard y C. Reavis, 2007) En lo referido al espacio de la V4 el año clave ha sido el 2005 cuando se firmó el acuerdo de adquisición de la totalidad de activos de la empresa británica Ready Mixed Concrete Limited (RMC) con instalaciones en la República Checa, Hungría y Polonia. La transacción marcó el fin de la compañía RMC y ofreció una fuerte posición de partida para CEMEX en tres países de la V4, pero sin completar la región por la ausencia de presencia de la RMC en Eslovaquia.

El acuerdo de compra consistía en una expansión repentina de CEMEX por la adquisición de activos en 20 países. Con esta adquisición CEMEX pretendía lograr cuatro objetivos. En primer lugar, CEMEX intentó convertirse en líder mundial en el sector de cemento, en segundo lugar pretendió diversificar el riesgo por medio de una mayor presencia internacional, en tercer lugar, aumentar su presencia en EE.UU. (a través de adquisición de las instalaciones de RMC en EE.UU.), mejorar su posicionamiento en el fuerte mercado de Europa Occidental y acceder a las economías de Europa del Este, que presentaban buenas expectativas de futuro crecimiento, por su desarrollo inmobiliario y de infraestructuras, y por último, mejorar su accesibilidad a la financiación por ser considerada una compañía con liderazgo en los mercados desarrollados. ${ }^{14}$

Una vez efectuado el acuerdo comercial las instalaciones polacas de RMC se rebautizan con el nombre CEMEX POLSKA. Actualmente CEMEX Polska tiene en su propiedad tres instalaciones cementeras, cuarenta plantas de hormigón, nueve canteras de piedra y en total emplea unos 1137 trabajadores.

Las prácticas de CEMEX en Polonia han causado dos grandes ecos en el país. En 2009 la Comisión Europea inició la investigación de un presunto caso de colusión entre siete empresas que operan en el mercado polaco de materiales de construcción. ${ }^{15}$ El Tribunal de Justicia de la Unión Europea descubrió que los orígenes de los acuerdos ilegales entre las empresas cementeras se remontan al año 1998. CEMEX en su defensa negó cualquier culpa o actuación de mala fe en el litigio, por no haber estado presente en el mercado polaco en el tiempo en que comenzó dicha práctica. A pesar de las objeciones por CEMEX y de otras compañías (tampoco presentes en Polonia a la fecha de 1998) la sentencia condenó CEMEX a pagar una multa de 27,9 millones de euros. Inmediatamente tras la resolución judicial CEMEX presentó un recurso en contra del fallo, por lo que la pena

\footnotetext{
${ }^{14}$ http://www.bloomberg.com/apps/news?pid=newsarchive\&refer=latin_america\&sid=ayEJbHnXryyc. Publica_ do: 27/09/2004. Consultado: 19/10/2015

15 http://sipse.com/negocios/sancionan-a-cemex-en-polonia-67806.html. Publicado: 26/12/2013. Consultado: $19 / 10 / 2015$
} 
Nedela, Daniel. Análisis de la presencia de las multilatinas en el espacio económico del grupo de Visegrád.

pecuniaria no ha sido liquidada y a principios de 2016 todavía se espera a la resolución definitiva de dicho pleito. ${ }^{16}$

También sobresale la presencia de CEMEX en Polonia por la excelencia en el proceso productivo y en la innovación medioambiental. En 2011 la planta de cemento en la ciudad Chelm es incluida en el Reglamento Comunitario de Ecogestión y Ecoauditoría (EMAS) por destacar en su compromiso en la conservación y preservación del medioambiente. ${ }^{17}$ En su momento la planta de Chelm formaba parte de un grupo reducido de 28 empresas instaladas en Polonia que han reunido los requisitos del Reglamento (EMAS). Una continua innovación productiva y medioambiental, unida a la aplicación de nuevas tecnologías que reducen las emisiones de $\mathrm{CO}_{2}$ en el proceso de fabricación de cemento en la planta de Chelm por lo que fue reconocida también por la revista Forbes con la entrega del premio "Líder de sostenibilidad" en el año 2012. ${ }^{18}$ A lo largo de su presencia en Polonia, CEMEX ha sido galardonado también por otros premios entre los cuales destacan el Hércules 2011, otorgado por el desarrollo de la industria de construcción, y el premio Águilas de la Construcción concedido por el uso efectivo de los fondos de la Unión Europea. ${ }^{19}$

En Hungría, la transacción de los activos del grupo RMC incluía la filial Danubiusbeton Betonkeszító, que posteriormente, pasa a denominarse en el año 2008 CEMEX Hungária para resaltar la presencia de la compañía mexicana en el país. ${ }^{20}$ A lo largo del mismo año CEMEX empezó a negociar la venta de sus instalaciones en Hungría y en Austria al grupo Strabag de origen austriaco. Al final el acuerdo no se materializó por la retirada de su socio en 2009. En respuesta CEMEX presentó una demanda judicial con reclamación del lucro cesante contra Strabag. En el año 2012 un tribunal arbitral en Europa declaró que la rescisión del contrato para la compra de los activos de CEMEX por parte de Strabag era ilegítima e inválida por lo que impuso una multa a Strabag de 37,1 millones de euros. ${ }^{21}$ Hoy en día CEMEX sigue estando presente en Hungría y según la publicación más reciente de su filial húngara, la compañía opera 34 plantas de hormigón y 5 canteras de piedra en el país.

En la República Checa el acuerdo de adquisición de los activos de RMC consistía en la compra de 4 compañías independientes administradas por la empresa británica (Ready Mix Bohemia, Transport Beton Morava, Readymix Praha - Malešice y CZ Sands). Una vez celebrado el acuerdo, a pesar de mantener las divisiones internas según el tipo de producto ofrecido, las cuatro empresas se unificaron bajo el mismo nombre de CEMEX Czech Operations, resaltando así la presencia de la compañía. Un cambio de nomenclatura igual al ocurrido en Hungría y con la misma motivación

\footnotetext{
16 http://eleconomista.com.mx/corporativos/2009/12/10/cemex-recibe-multa-millonaria-polonia Publicado: 10/12/2009 Consultado: 19/10/2015

17 http://www.cemex.com/MediaCenter/Story/Story20111031_4.aspx Publicado: 31/10/2011 Consultado: $19 / 10 / 2015$

18 http://www.cemex.com/MediaCenter/Story/Story20120809.aspx Publicado: 09/09/2012 Consultado: 19/10/2015

${ }^{19}$ http://www.cemex.com/ES/SalaDePrensa/Historia/Historia20131127.aspx Publicado: 27/11/2013 Consultado: $19 / 10 / 2015$

${ }^{20} \mathrm{http}: / /$ satellite.tmcnet.com/news/2007/05/03/2582018.htm Publicado: 03/05/2007 Consultado: 19/10/2015

${ }^{21} \mathrm{http}: / /$ www.excelsior.com.mx/2012/06/11/dinero/840581 Publicado: 06/11/2012 Consultado: 19/10/2015
} 
Nedela, Daniel. Análisis de la presencia de las multilatinas en el espacio económico del grupo de Visegrád.

de crear poder para la marca CEMEX. Con esta transacción la multinacional mexicana accedió al mercado checo como el segundo fabricante de hormigón (con 76 instalaciones), tercero en la fabricación de cemento (con 2 instalaciones) y cuarto en la producción de piedra. ${ }^{22}$

En el año 2013, CEMEX completa su presencia en la V4 con la extensión de su actividad a la República Eslovaca. La división CEMEX Sands, originaria de la CEMEX Czech Operations, concluye un acuerdo de cooperación estratégica a largo plazo con la Empresa de Construcción de Carreteras de la ciudad de Žilina en Eslovaquia. ${ }^{23}$ A la diferencia de la experiencia previa de CEMEX, basada en una fuerte entrada al mercado foráneo, su actividad en Eslovaquia ha sido emprendida de un modo diferente. El acuerdo de cooperación incluyó la venta de 3 canteras de piedra a CEMEX que en sí mismas no garantizan una elevada cuota en el mercado. A pesar de no conquistar con ello el mercado eslovaco, el acuerdo tiene un gran valor comercial dado que en los alrededores de Žilina se encuentra el último tramo que todavía queda por construir de la carretera que recorre de oeste a este el país. El proyecto del tramo que falta ya está diseñado en su totalidad y es considerado de elevada importancia a nivel europeo. Para la construcción de dicho intervalo entre un $60 \%$ y $70 \%$ del material necesario se podrá obtener de las instalaciones extractivas de CEMEX en Žilina. La localización de CEMEX puede jugar un rol importante en este proyecto por su competitividad vía precio debido a la cercanía del lugar de producción de piedra y de construcción.

En el año 2014 CEMEX Czech Operations es galardonado por la consultora AON Hewitt con el premio del Mejor Empleador en la categoría de grandes empresas. El premio fue otorgado por ser una de las tres empresas que obtuvieron la mayor puntuación en 5 indicadores (motivación, satisfacción con la dirección de la empresa, productividad, capacidad de atraer nuevos empleados y retención de empleados así como el deseo de los mismos de mantener relaciones con la empresa en el futuro). ${ }^{24}$

EL último gran cambio que se ha producido en el escenario de la V4 respecto a la presencia de CEMEX tuvo lugar en el año 2013, cuando empezaron las negociaciones con su rival Holcim. Las negociaciones culminaron con uno de los mayores acuerdos de adquisición y fusión en los últimos años. En lo que se refiere a CEMEX, este acuerdo le permite fortalecer su posición en la República Checa a cambio del cese de sus activos en Alemania occidental, pero manteniendo su actividad en el conjunto del país. A su vez el acuerdo contempla una alianza de cooperación entre ambas compañías en España donde el total de las ventas se distribuirá de forma que $75 \%$ permanecerá en CEMEX y del $25 \%$ restante se beneficiará Holcim. A través de esta operación CEMEX intenta mejorar su valor en el indicador EBITDA (según las estimaciones oficiales en unos 20-30 millones de dólares por año), aprovechar el contexto macroeconómico favorable y las expectativas de crecimiento de la demanda en la República Checa y una mayor adaptabilidad al mercado español unida a una revalorización de sus activos en dicho

\footnotetext{
${ }^{22}$ http://www.betontks.cz/casopis/BETON_TKS_2007-01.pdf Publicado: 16/02/2007 Consultado: 19/10/2015

${ }^{23} \mathrm{http}: / / w w w . c e m e x . c z / c e m e x-v s t u p u j e-n a-s l o v e n s k o . a s p x$ Publicado: 25/04/2013 Consultado: 19/10/2015

$24 \mathrm{http}: / /$ www.cemex.cz/cemex-dostal-oceneni-best-employer-2014.aspx Publicado: 28/11/2014 Consultado: $19 / 10 / 2015$
} 
Nedela, Daniel. Análisis de la presencia de las multilatinas en el espacio económico del grupo de Visegrád.

mercado. La transacción recibió visto bueno del departamento antimonopolio de la Comisión Europea y concluyó con la fecha de 5.1.2015. ${ }^{25}$ Por las transferencias realizadas en República Checa, Alemania y España, Holcim abonó 70 millones de euros a CEMEX. ${ }^{26}$

\subsection{NEMAK - Garantía de suministro seguro}

NEMAK es una empresa de origen mexicano que desde el año 1979 forma parte del Grupo ALFA. A diferencia de las demás empresas multinacionales procedentes de América Latina presentes en la región de la V4, el grupo ALFA se centra en una variedad de actividades. El grupo ALFA es la matriz de unas 5 empresas cuya actividad abarca diferentes sectores, desde la alimentación hasta la petroquímica y además dispone de participaciones en otras empresas ajenas a su estructura básica. ${ }^{27}$ La estrategia seguida por el Grupo es la de minimizar el riesgo por medio de una diversificación de actividad económica y no exclusivamente a través de expansión geográfica como ocurre en el caso de CEMEX. La lógica que está detrás de este tipo de actuación se sustenta en la idea de que unas posibles pérdidas en una determinada actividad económica se complementan con ganancias en otros sectores. La empresa NEMAK es un tentáculo del Grupo ALFA cuyo proceso productivo se ubica dentro de la industria automotriz, concretamente en la fabricación de los componentes de aluminio del bloque motor.

La relevancia de la experiencia de NEMAK para la región radica en su estrategia de entrada al espacio de la V4. NEMAK no cumple con los supuestos que establecen el modo de entrada de las multilatinas a un merado nuevo se produce por medio de las fusiones $o$ adquisiciones. En el caso de NEMAK, la primera expansión hacia el mercado checo se produce por una inversión greenfield y está orientada hacia la búsqueda de mercados y de las ventajas que ofrece la localización en el centro de la Unión Europea, junto con otras características propias de los países poscomunistas que a su vez se encuentran cerca de los mercados nacionales más potentes del continente. Debido a esto NEMAK emprende sus primeros pasos de la expansión internacional hacia V4 en la República Checa, que a su vez comparte la frontera con Alemania.

Si nos centramos en las ventajas que proporciona la localización en la región de V4 la entrada de NEMAK se puede explicar por el Paradigma "OLl" de Dunning (J. H. Dunning, 1994, 1999). Siendo NEMAK una empresa siderúrgica especializada en la fabricación de componentes de aluminio para la industria automotriz y dentro de esta rama en la fabricación de los motores cilíndricos, la expansión internacional a la V4 es justificada por una elevada presencia de grandes marcas de fabricantes de automóviles. NEMAK identificó a ocho compañías automovilísticas como claves para su existencia y creación del beneficio entre las cuales seis disponen de líneas de producción en la región de V4 y otras dos están localizadas en los alrededores más

\footnotetext{
${ }^{25}$ Diario Oficial de la Unión Europea (2014/C438/09), http://eur-lex.europa.eu/legal-content/EN/TXT/PDF/ ?uri=CELEX:52014XX1206(02)\&qid=1438677746616\&from=EN

26 http://www.cemex.sk/cemex-zlepsuje-svoju-poziciu-v-europe.aspx\#237; ciuvEurand Publicado: 28/09/2013 Consultado: 19/10/2015

27 http://www.alfa.com.mx/NC/perfil.htm Publicado: PÁGINA OFICIAL DEL GRUPO SIN FECHA DE PUBLICACIÓN Consultado: 19/10/2015
} 
Nedela, Daniel. Análisis de la presencia de las multilatinas en el espacio económico del grupo de Visegrád.

cercanos. $^{28}$ A su vez la producción de NEMAK no está dedicada en exclusiva al suministro a las compañías mencionadas anteriormente, sino que, también funciona como proveedor para otras marcas automovilísticas que también se encuentran en el espacio de Europa Central ${ }^{29}$.

El modo de entrada de NEMAK a la República Checa tampoco ha seguido las pautas habituales de la expansión internacional de las multilatinas. De modo general, las empresas procedentes de Latinoamérica prefieren entrar en otros mercados por medio de fusiones y adquisiciones de empresas ya existentes que son dominantes en el espacio que es objeto de su expansión o por medio de Joint Ventures con compañías locales. A la diferencia de la tendencia observada, el primer tipo de penetración hacia V4 por parte de NEMAK ha sido una inversión greenfield con un valor aproximado de 30 millones de dólares. ${ }^{30}$

En el año 2003, tras las negociaciones entre los representantes de NEMAK y las autoridades competentes de la República Checa, se aprobó el plan para la construcción de una planta en la ciudad de Most localizada en el oeste del país. Las razones para invertir en la República Checa, como indica la propia empresa en sus comunicados son: baja presión fiscal; garantía de la protección estatal de la propiedad; alta productividad y mano de obra cualificada y barata respecto a la media de la UE ${ }^{31}$.

Tras haber realizado la primera inversión en la V4, NEMAK expande su actividad a los países vecinos de su nueva economía host en Europa, esta vez ya siguiendo los pasos acordes a la tendencia general de la expansión internacional de las multilatinas.

Cronológicamente la segunda expansión de NEMAK en la región de V4 tuvo lugar en el año 2004 y fue dirigida hacia Alemania y Eslovaquia. La entrada a estos mercados se realizó por medio de la adquisición de la empresa alemana denominada Rautenbach-Aluminium-Technologie.

En lo que concierne a los activos de Rautenbach-Aluminium-Technologie localizados en Eslovaquia, éstos se encuentran en la ciudad de Žiar nad Hronom que cuenta con una larga historia relacionada con la producción y procesamiento de aluminio. Las razones que condujeron a NEMAK hacia la adquisición de la empresa alemana ha sido su posición estratégica en el mercado europeo. En concreto, respecto a las instalaciones en Eslovaquia los representantes de NEMAK valoraron positivamente una alta productividad de la región así como baja conflictividad laboral y el bajo

\footnotetext{
${ }^{28}$ Fiat - Chrysler (Polonia), Ford (Hungría), GMC (Polonia), Hyundai - KIA (Eslovaquia), Volkswagen (Eslovaquia y República Checa), BMW (Austria y Alemania) y Renault - Nissan (Eslovenia)

${ }^{29}$ Peugeot - Citroën (Eslovaquia, República Checa), Toyota (Polonia, República Checa), Suzuki (Hungría), Daimler (Hungría), Jaguar - Land Rover (la construcción de la planta fue negociada entre la compañía y los representantes del gobierno eslovaco a lo largo de la primera mitad del año 2015 y la decisión de construir una nueva planta de producción de JLR ha sido tomada a principios de agosto de 2015)

30 http://archiv.ihned.cz/c1-821739-nemak-investuje-v-cr-miliardu Publicado: 26/11/1999 Consultado: $19 / 10 / 2015$

${ }^{31}$ Según los datos relativos al año 2014, el coste laboral medio en la República Checa es igual a 9,4 euros por hora trabajada, muy por debajo de la media de la UE situada en 24,6 euros (el séptimo más bajo de la UE). fuente: http://ec.europa.eu/eurostat/tgm/table.do?tab=table\&init=1\&language=en\&pcode=tps00173\&plugin=1 Publicado: 02/03/2015 Consultado: 19/10/2015
} 
Nedela, Daniel. Análisis de la presencia de las multilatinas en el espacio económico del grupo de Visegrád.

coste de establecimiento y funcionamiento. Es decir, que se trata de una paráfrasis de los motivos que condujeron a su expansión hacia la República Checa en el año anterior. ${ }^{32}$

La expansión a Eslovaquia trajo consigo su fruto más destacable ya durante el año de entrada, cuando NEMAK obtuvo el contrato de suministro único de motores de cuatro cilindros para KIA Motors Company que empezó su producción a finales de 2006. El contrato contenía también las cláusulas relativas al aumento de producción de la planta automovilística hasta alcanzar el pleno rendimiento en el año 2009 con aproximadamente 300.000 vehículos al año y de forma sincronizada al incremento extendía el suministro exclusivo de NEMAK para la totalidad de la producción. La experiencia de NEMAK en Eslovaquia ha sido muy productiva lo que demuestra la entrega del Premio de la República Eslovaca a la Calidad en el año 2013 y también la colaboración entre la empresa y las universidades técnicas localizadas en áreas cercanas. $^{33}$

En agosto de 2015 NEMAK anunció una ampliación de producción en la planta eslovaca con una inversión de 8,75 millones de euros. La extensión de la planta creará aproximadamente 100 puestos de trabajo y un $60 \%$ de la producción estará destinado a la exportación.

La tercera expansión de NEMAK en V4 fue dirigida hacia Hungría y también como en el caso de Eslovaquia se produjo por medio de una adquisición. En el año 2006 NEMAK adquiere cuatro plantas de producción y procesamiento de aluminio de la empresa noruega Norsk Hydro por un valor de 552,6 millones de dólares. El motivo de la expansión sigue en la línea anterior mencionando las ventajas de la mano de obra cualificada, cercanía a centros industriales y se añade de manera explícita la existencia de buenas infraestructuras que facilitan la logística en el proceso de suministro a los clientes. ${ }^{34}$

La sucursal húngara de la compañía NEMAK ha logrado obtener unos beneficios excepcionales que rondaron 150 millones de dólares en el año 2013 a raíz de lo que en el año posterior se decidió realizar una expansión de las instalaciones existentes por un valor de 17,8 millones de euros a los que se uniría el subsidio de aproximadamente 1,25 millones de euros proporcionado por el gobierno húngaro acorde a su plan estratégico de reindustrialización del país. La culminación del proceso de ampliación está prevista para el año 2017 y según las estimaciones oficiales convertiría a Hungría en el país europeo que más aluminio procesa. Esta ampliación creará 100 nuevos puestos de trabajo directos que se unirán a los 798 ya existentes. ${ }^{35}$ Dentro de las actividades recientes de la sucursal húngara de NEMAK destaca también la firma del acuerdo de suministro único con Renault-Nissan que entrará en vigor a partir del año 2017 cuyo valor asciende a unos 200 millones de euros.

\footnotetext{
32 http://www.alfa.com.mx/down/2005/january31\%20_2005.pdf Publicado: 31/01/2005 Consultado: 19/10/2015 33 http://www.teraz.sk/ekonomika/narodnu-cenu-kvality-ziskali-nemak-d/28487-clanok.html. Publicado: 12/11/2012. Consultado: 19/10/2015

34 http://business.highbeam.com/437192/article-1G1-155580105/hungary-industry-norsk-hydro-sells-europeanaluminium Publicado: 6/12/2006 Consultado: 19/10/2015

35 http://www.kormany.hu/en/ministry-of-foreign-affairs-and-trade/news/huf-5-billion-factory-expansion-atnemak-gyor-kft Publicado: 04/09/2014 Consultado: 19/10/2015
} 
Nedela, Daniel. Análisis de la presencia de las multilatinas en el espacio económico del grupo de Visegrád.

NEMAK ha completado su expansión por los países de la V4 con la adquisición de siete plantas de la empresa italiana Teksid Aluminium por un valor de 485 millones de dólares y un 6,68\% del beneficio futuro de la empresa a Teksid. De las siete plantas seis están localizadas en el continente americano y una en la ciudad Bielsko-Biała en el sur de Polonia. Desagregando el acuerdo, la compra de la planta de procesamiento de aluminio en Polonia costó 56,1 millones de dólares, más un reparto de beneficios procedentes de la planta en valor de 0,83\%. ${ }^{36}$ En el año 2012 NEMAK Polska controla un $11 \%$ de toda la industria metalúrgica del país (incluido el procesamiento de hierro, acero, cobre y aluminio).

\subsection{KATCON - el arte de ser mundial de un día a otro}

KATCON Global es una empresa fundada en el año 1993 que forma parte del grupo Bienes Turgon procedente de México. Igualmente como en el caso del grupo ALFA (también presente en la V4 con NEMAK) su actividad está diversificada en varias áreas del mercado, desde el sector alimenticio hasta el sector inmobiliario y automovilístico. Respecto a la industria automotriz, Bienes Turgon es la casa matriz de KATCON, una empresa cuya actividad se especializa en la fabricación de los tubos de escape, que desde el año 2009 tiene presencia internacional. La expansión a los mercados foráneos por parte de KATCON tiene su origen en el año 2009 con la adquisición de la compañía estadounidense Delphi por Bienes Turgon. En el año 2014 KATCON diversifica su actividad hacia el sector de la fabricación de componentes de peso ligero para la industria automotriz y de aeronáutica a base de la fibra de carbono. ${ }^{37}$

El acuerdo de compra contemplaba la totalidad de negocios relativos a la fabricación de los tubos de escape de Delphi a nivel mundial, lo que proporcionó a KATCON una posición fuerte en el mercado y junto con el traspaso de tecnología y de conocimiento ofreció una buena base para poder mantener y fortalecer este posicionamiento. El acuerdo de venta en su fase inicial incluyó el trasvase de los derechos de propiedad intelectual, patentes, innovaciones en procesos de producción, junto con 6 plantas de producción y dos centros de investigación. Una de estas plantas se encuentra en Polonia, en la ciudad de Błonie, y depende del centro de planificación estratégica localizado en Luxemburgo (igualmente procedente del grupo Delphi). La segunda fase del acuerdo de compra cuenta con el traspaso de unidades productivas localizadas en India y China por lo que KATCON se convertirá en una empresa con presencia global. ${ }^{38} \mathrm{El}$ objetivo perseguido por KATCON a la hora de su expansión internacional era ganar acceso a los mercados desarrollados y al mismo tiempo penetrar en los mercados emergentes con mayor potencial de crecimiento y poder acomodar la producción a las altas demandas estimadas de proveedores de la industria automotriz en estas regiones. ${ }^{39}$

\footnotetext{
${ }^{36}$ http://foundrymag.com/uncategorized/nemak-closes-purchase-teksid-aluminum-poland Publicado: 19/04/2007 Consultado: 19/10/2015

37 http://www.katcon.com/advancedmaterials/wp-content/uploads/2015/04/KATCON_BrochureDigital.pdf Publicado: DOCUMENTO DE KATCON SIN FECHA DE PUBLICACIÓN Consultado: 19/10/2015

${ }^{38} \mathrm{http} / / / \mathrm{www}$.prnewswire.com/news-releases/bienes-turgon-and-katcon-announce-the-closing-of-the-first-stageof-the-acquisition-of-delphis-global-exhaust-system-business-61764102.html Publicado: $05 / 05 / 2009$ Consultado: 19/10/2015

39 http://eleconomista.com.mx/industrias/2011/01/07/regia-katcon-abre-escape-su-expansion Publicado:
} 07/01/2011 Consultado: 19/10/2015 
Nedela, Daniel. Análisis de la presencia de las multilatinas en el espacio económico del grupo de Visegrád.

La filial polaca, KATCON Polska, se encuentra a fueras de la capital Warsovia y sus principales áreas de actividad son la fabricación de tubos de escape para la industria automotriz. Siendo ésta la única planta de producción de KATCON localizada en Europa su mercado se extiende desde Portugal hasta Turquía. Durante sus años de funcionamiento en Polonia, KATCON valora muy positivamente la interconexión viaria y ferroviaria de la región, lo que en gran medida facilita la logística para la venta. ${ }^{40}$ La estrategia seguida en el proceso de la multinacionalización de KATCON ha sido la búsqueda de nuevos mercados, desarrollo de la producción modular, acceso a las tecnologías sofisticadas y a los procesos de producción innovadores. ${ }^{41}$ Se puede identificar un cierto paralelismo entre los motivos de atracción hacia la V4 por parte de KATCON y NEMAK, dado que las dos empresas destacaron las ventajas de la mano de obra flexible y comparativamente barata que puede suministrar a los mercados con mayor capacidad adquisitiva.

\subsection{Stefanini IT Solutions ${ }^{42}$ - el mundo es pequeño gracias a las TIC}

Stefanini IT Solutions es una empresa de origen brasileño que se especializa en los servicios informáticos y de outsourcing. La fundación de la empresa se remonta al año 1987 y su conversión en una compañía multinacional ocurre en el año 1996 con su primera expansión internacional hacia Argentina. Un liderazgo ambicioso unido a la especialización en la provisión de servicios en campos como telecomunicaciones, finanzas y recursos energéticos ha permitido que Stefanini IT Solutions se convierta en una empresa con presencia global, que a fecha de 2015, suma unos 33 países y alrededor de 80 oficinas. La rápida expansión de la empresa es una demostración de cómo los adelantos tecnológicos impulsan la integración de los mercados nacionales en la economía mundial y aceleran el proceso de globalización.

La llegada de Stefanini IT Solutions hacia la V4 se produce en el año 2006 y según las palabras de sus directivos, los motivos para el establecimiento en Polonia se encuentran en una mano de obra cualificada y además ven ventajas intrínsecas en el idioma polaco y su elevado grado de similitud con los demás idiomas eslavos, así como en el tamaño de mercado del país. A lo largo del año 2014 la empresa amplió su presencia en la región con la apertura de una oficina en Hungría. Cabe destacar que las oficinas localizadas en estos dos países prestan servicios más allá de las fronteras nacionales y de este modo fortalecen la posición de la compañía en Europa lo que demuestra también su amplia oferta lingüística de atención al cliente. ${ }^{43}$

Cabe mencionar, que la expansión internacional de empresas dedicadas al sector de servicios sigue unas pautas diferenciadas respecto a las compañías industriales. En este caso el capital financiero exigido para la expansión internacional es comparativamente inferior y además el espacio de prestación de servicios en la Era

\footnotetext{
${ }^{40}$ http://www.katcon.com/facilities/poland/ Publicado: PÁGINA OFICIAL DE KATCON SIN FECHA DE PUBLICACIÓN Consultado: 19/10/2015

41 http://www.katcon.com/about-us/ Publicado: PÁGINA OFICIAL DE KATCON SIN FECHA DE PUBLICACIÓN Consultado: 19/10/2015

${ }^{42}$ Siendo poco habitual el proceso de transnacionalización de las empresas multilatinas dedicadas a los servicios no industriales, el caso de Stefanini IT Solutions se menciona para completar el panorama de la presencia latinoamericana en la V4.

${ }^{43}$ https://www.youtube.com/watch?v=B7D7-sPd4jw Publicado: 24/04/2013 Consultado: 03/02/2015
} 
Nedela, Daniel. Análisis de la presencia de las multilatinas en el espacio económico del grupo de Visegrád.

de la Información no conoce de barreras nacionales. En este aspecto, Stefanini IT Solutions destaca por su orientación hacia la excelencia reflejada tanto en la retención de clientes con contratos de larga duración que supera un 90\% como en la retención del talento igualmente situada en un $90 \% .{ }^{44}$ Estas estadísticas confirman los resultados obtenidos por la consultora Towers Watson en una encuesta sobre la gestión del capital humano, según cual las multilatinas mencionan esta variable como determinante a la hora de desarrollar su actividad. (Towers Watson, 2010)

\section{RESULTADOS}

Tras haber realizado un recorrido por las trayectorias de las cinco multilatinas más importantes en el espacio de la V4 procedemos a la identificación más detallada de los factores comunes que motivaron su internacionalización y a su vez intentaremos aplicar el marco teórico a este proceso.

Empezando con la dimensión temporal de la expansión internacional de las multilatinas podemos confirmar las observaciones de Casanova (L. Casanova, 2010) que fijan la inserción de las multilatinas al escenario de la economía mundial a partir del año 2002. Todas las incursiones de las multilatinas en la V4 han tenido lugar con posterioridad a esta fecha; empezando con NEMAK en el año 2003, seguido por CEMEX en el 2005, Stefanini IT Solutions en el 2006, KATCON en el 2009 y Embraer dos años más tarde. Desde el punto de vista de relaciones de comercio y de inversión observamos que las multilatinas se convierten en jugadores globales precisamente en el momento en que entran en mercados poco conocidos, como son los de los países de la V4. A su vez, hay que tener en cuenta un dato crucial que está detrás de esta internacionalización, que es su particular modo de entrada, efectuado por medio de fusiones y adquisiciones con otras empresas multinacionales. Dos de los cinco casos analizados han empleado esta táctica a la hora de instaurarse en el mercado de la V4. Solamente la empresa NEMAK emprendió su llegada a la región por medio de una inversión greenfield en República Checa en el año 2003, siendo esta la primera experiencia de una empresa latinoamericana en la región analizada. A su vez Embraer tampoco ha optado por esta estrategia, su entrada se realizó bajo un acuerdo de riesgo compartido, por medio de una Joint Venture. Exceptuando estos dos casos del primer contacto con el territorio de la V4 hay que mencionar que la progresiva expansión por la región confirma la hipótesis de la entrada de las multilatinas por medio de fusiones y adquisiciones, más bien estas últimas, en todos los demás casos.

El modo de entrada de NEMAK a República Checa por medio de una inversión greenfield demuestra el interés directo de la compañía mexicana en la región, pero en los demás casos la entrada se efectúa por medio de una adquisición de empresas multinacionales con activos en varios países y no exclusivamente en la región de la V4. De este modo NEMAK profundiza su presencia en la V4 por medio de la adquisición de Rautenbach-Aluminium-Technologie, de origen alemán, y de Norsk Hydro, originalmente de Noruega. CEMEX efectúa su entrada por la adquisición de Ready Mix Concentrate, procedente de Reino Unido y KATCON la realiza a través de la adquisición de Delphi, originaria de EE.UU.. A base de esta 44 http://www.stefanini.ro/company/why-stefanini-adc Publicado: PÁGINA OFICIAL DE KATCON SIN
FECHA DE PUBLICACIÓN Consultado: 03/02/2015 
Nedela, Daniel. Análisis de la presencia de las multilatinas en el espacio económico del grupo de Visegrád.

observación podemos decir que el motivo que ha dado lugar a la primera expansión al mercado de la V4 no se encuentra exclusivamente en lo que es la misma región, sino que hay que buscarlo en las características del negocio de las empresas adquiridas. Siendo todas las empresas adquiridas procedentes de las economías desarrolladas, por detrás de estas características se encuentran motivos relacionados con el acceso a la tecnología o la conquista de nuevos mercados para obtener posibilidades de crecimiento orgánico de la empresa.

Aplicando las teorías que correlacionan el nivel del desarrollo con el flujo de la IED elaboradas por Lucas (R. E. Lucas, 1990) y Cuervo-Cazurra (Á. Cuervo-Cazurra, 2008, 2010) analizamos las características principales de los países latinoamericanos emisores de la IED. La región de emisión es bastante amplia, pero en la V4 encontramos solamente la presencia de empresas con origen en Brasil y México. En primer lugar, apoyándose en R. Lucas, preguntamos el ¿por qué? de la inversión procedente de unos países con un nivel de desarrollo inferior al de la V4 y con unos costes más elevados. Las razones de esta inversión se hallan en la cercanía de los grandes mercados europeos, en una buena red de infraestructuras, en una alta cualificación de la mano de obra y en la localización dentro del espacio de la Unión Europea. Estos factores, al fin y al cabo, compensan los costes asociados con la entrada en unos mercados más caros. A su vez, desde la consolidación del nuevo régimen a partir del año 1989 y gracias al apoyo de las economías más potentes de la Unión Europea, la región de la V4 presenta una evolución de los indicadores macroeconómicos positiva que proporciona ventajas intrínsecas relacionadas con el robustecimiento de la estabilidad institucional.

Examinando las características comunes de los países emisores de la IED latinoamericana observamos que México y Brasil forman parte de la primera categoría en la clasificación de Cuervo-Cazurra que correlaciona las características económico-institucionales con el flujo de salida de la IED. Son países con mucha población y un mercado potente que permite la creación de unas FSA suficientes para la expansión internacional. No es sorprendente encontrar la presencia de las empresas de los países mencionados dado que la mayoría de las multilatinas tiene su origen en uno de los tres países latinoamericanos económicamente más internacionalizados (México, Brasil y Chile). Según el Ránking de las 100 Multilatinas, elaborado por la AméricaEconómica Intelligence, 34 son procedentes de Brasil, 25 de México y 17 de Chile (aunque este último país, a fecha de hoy, no está presente con una compañía relevante en la V4). ${ }^{45}$

Respecto a la teoría The bridge model (L. Casanova y E. Rodríguez-Montemayor, 2014) podemos observar el cumplimiento de la tendencia en el proceso de transnacionalización de NEMAK y de CEMEX. Las dos empresas se expandieron primero a España y posteriormente siguieron con la estrategia de conquistar el mercado europeo, en sus respectivos sectores, por medio de inversiones greenfield o fusiones y adquisiciones con compañías presentes en los demás países de la UE. Igualmente en el caso de Embraer la teoría se cumple con la adquisición de acciones de la empresa portuguesa OGMA en el año 2005, siendo esta su fecha de

\footnotetext{
${ }^{45}$ http://rankings.americaeconomia.com/multilatinas-2014/ranking/ Publicado: ÁMERICA ECONÓMICA SIN FECHA DE PUBLICACIÓN Consultado: 19/10/2015
} 
Nedela, Daniel. Análisis de la presencia de las multilatinas en el espacio económico del grupo de Visegrád.

entrada al territorio europeo ${ }^{46}$ En lo referido a KATCON, su trayectoria no coincide con la tendencia general de la teoría del puente iberoamericano. Las únicas instalaciones del grupo Bienes Turgon en Europa son KATCON Polska y KATCON R\&D Center Luxembourg. La presencia de KATCON en Polonia es anómala dado que Bienes Turgon estaba interesado en la adquisición de la totalidad de los activos de Delphi incluidos los patentes, innovaciones en los procesos de producción y centros de investigación. Dada la presencia mundial de Delphi y las patentes registradas bajo su nombre, el motivo de la adquisición se encuentra en el proceso de la innovación revertida y en la conquista del mercado mundial y no en un interés exclusivo en la V4. El modo de entrada de las empresas seleccionadas a la Unión Europea, y a la V4 en particular, coincide con los datos presentados en un informe de Towers Watson que en el primer lugar identifica el uso de la herramienta de fusiones y adquisiciones como la preferida (55\% de expansiones), en segundo lugar se encuentra una inversión greenfield (26\%) y en tercer lugar el establecimiento de una Joint Venture (19\%). (Towers Watson, 2013)

Desde el punto de vista de A. M. Rugman (A. M. Rugman, 1981) y de su modelo basado en las FSA y CSA se confirma la presencia de las FSA en los cuatro casos de empresas industriales estudiadas, además en el caso de Embraer y CEMEX están presentes también las CSA. Siendo las dos multinacionales surgidas a partir de campeones nacionales. El robustecimiento de las FSA de las cuatro compañías se ha construido en torno a la imagen de la marca como garantía de productos de alta calidad. Las FSA conseguidas de este modo permitieron a las empresas acumular una cantidad suficiente de recursos que se han utilizado para su expansión internacional a nivel global a través de adquisiciones o inversiones greenfield, que a su vez están retroalimentando las FSA originarias. El robustecimiento de las FSA se justifica con una presencia internacional cada vez más grande de estas empresas.

Otro marco teórico aplicable a la presencia de las multilatinas es el Paradigma OLI de Dunning (J. H. Dunning, 1994). Dado que es una herramienta de investigación aplicable para todos los procesos de transnacionalización de las empresas, se identificarán los principales motivos que se encuentran por detrás de cada una de las siglas que le dan nombre al paradigma. Bajo el factor "localización" nos encontramos con una pléyade de elementos que actúan en favor de la región de la V4. Empezando desde lo macro podemos aludir a una ya mencionada evolución positiva de los indicadores macroeconómicos en los cuatro países de la V4. La crisis económica del 2007 no ha afectado al crecimiento de la región como lo ha hecho en otros países europeos. Independientemente de la organización encargada de realizar el pronóstico, la V4 mantiene una previsión sobre el futuro crecimiento bastante buena, superior a 2,5\% del PIB (véase Banco Mundial, Bloomberg, Comisión Europea, Economist Intelligence Unit). Entre otras ventajas presentes en la región se encuentra, por un lado, la presencia de una mano de obra cualificada y respecto a los estándares de la UE relativamente barata y por el otro, una eficiente red de infraestructuras que conecta estos países con mercados cercanos de altos ingresos y una demanda creciente. De hecho, las cinco empresas analizadas han

\footnotetext{
${ }^{46}$ Oficinas Gerais de Material Aeronáutico (OGMA) era una empresa de propiedad pública hasta el año 2005 cuando se produjo la privatización de un $65 \%$ de sus acciones que fueron adquiridas por Embraer. Los $35 \%$ restantes siguen en la propiedad de la República Portuguesa.
} 
Nedela, Daniel. Análisis de la presencia de las multilatinas en el espacio económico del grupo de Visegrád.

destacado la ventaja de unos costes laborales inferiores a la media europea de la V4. En los casos de Embraer, KATCON Y NEMAK podemos incluir también la posibilidad de integración en las Cadenas Globales de Valor de la industria automotriz y aeronáutica, las dos muy presentes en la región, y la probabilidad de un spillover tecnológico, dado el nivel científico que proporciona la localización en la UE. Los factores de atracción localizados en la V4 coinciden con los motivos que conllevan a las multilatinas hacia la expansión internacional. En una encuesta de Towers Watson realizada sobre una muestra de las multilatinas más influyentes en el mundo los tres motivos principales para la entrada a mercados foráneos son: la búsqueda de nuevos mercados (citado por el 100\% de las empresas encuestadas), integración en las CGV (30\%) y el acceso a tecnología (15\%). (Towers Watson, 2013)

Entre las ventajas proporcionadas por el segundo elemento del Paradigma OLI, "propiedad", según las declaraciones de los directores generales de KATCON y Embraer, de nuevo encontramos presente el factor tecnología. Además las multilatinas que han optado por una entrada por medio de fusiones y adquisiciones destacan la importancia de la posición en el mercado ostentada por la compañía adquirida. Una elevada cuota de mercado permite a las multilatinas establecidas en la región obtener ingresos significativos y al mismo tiempo, en el caso de que se produjeran ventas suficientes, fomentar el deseo de una ampliación de la inversión original; un proceso que ocurrió con CEMEX en Republica Checa y con NEMAK en los cuatro países de la región. A su vez, esta elevada rentabilidad de la inversión mejora los indicadores financieros de las empresas y les facilita el acceso a la financiación. El éxito de los negocios latinoamericanos en la V4 confirma la hipótesis relativa a las posiciones de liderazgo mundial de estas empresas. Como argumentos a favor de esta afirmación sirve con mencionar su presencia en una región altamente competitiva que se localiza en el mercado único de la UE y además con capacidad para desafiar a las empresas multinacionales originarias del mundo desarrollado sin poner en peligro su propia cuota de mercado. Por último, entre las ventajas de la internacionalización, encontramos los factores que son resultantes de los anteriormente mencionados y además podemos añadir las nuevas experiencias adquiridas con el proceso de internacionalización en las economías post-comunistas y, exceptuando Hungría, en la cultura empresarial eslava. En el año 2013, NEMAK ha utilizado con éxito los conocimientos adquiridos en la V4 para negociar su entrada al mercado ruso. La expansión se efectuó por medio de una inversión greenfield en la ciudad Kaluga, localizada en las cercanías de Moscú, donde se ubica la principal planta de producción de Volkswagen para dicho mercado. La factoría empezó con la producción en abril de 2015 y ha sustituido las vías anteriores de suministro basadas en el transporte internacional por una distribución directa in situ.

Por último, en lo referido a la Escuela de Uppsala, podemos establecer que a grandes rasgos, hay elementos que se cumplen pero no bastan para confirmar la aplicabilidad de la teoría para el caso de las multilatinas en la V4. Dada la diferencia cultural existente entre la V4 y América Latina, las multilatinas deberían estar escasamente presentes en la región estudiada lo que se cumple, pero la adquisición de RMC Group por parte de CEMEX o de Delphi por parte de Bienes Turgon no están bien justificadas a primera vista por esta teoría. Las experiencias de CEMEX y de KATCON no cumplen con las premisas establecidas por la Escuela de Uppsala y 
Nedela, Daniel. Análisis de la presencia de las multilatinas en el espacio económico del grupo de Visegrád.

su entrada a la V4 se explica con motivos que se escapan a la hipótesis de la teoría sueca. Las dos empresas se rigieron por la estrategia de conquistar el mercado mundial, en sus respectivas ramas, por medio de adquisiciones de jugadores importantes y no obedecieron ni los pasos de transnacionalización ni los elementos de proximidad cultural. Embraer tampoco ha perseguido una motivación basada en los motivos de cercanía o una previa presencia de puntos de venta. La estrategia del fabricante brasileño consistía en el acceso a la tecnología, para poder experimentar un proceso de innovación a la inversa con su socio más experimentado, y al mismo tiempo en el aprovechamiento de un coste relativamente bajo en comparación con los gastos que supondría el establecimiento de una Joint Venture con fabricantes de los países occidentales. En el caso de NEMAK la situación es más complicada. NEMAK entra al espacio de la V4 por medio de una inversión greenfield pero fijándonos en la actividad de esta empresa podemos encontrar rasgos que otorgan cierta validez a la Escuela de Uppsala. NEMAK ya con su primera expansión europea hacia España, ha conseguido suministrar las cabezas de motores de cuatro cilindros a la empresa alemana Volkswagen. Una posterior expansión de su actividad a la República Checa se puede justificar con la presencia de Volkswagen en dicho país. De este modo, NEMAK pudo aprovechar sus conocimientos de la cultura empresarial de Volkswagen, adquiridos durante una colaboración previa, y desde el comienzo de la producción de NEMAK en la República Checa pudo suministrar productos a su cliente ya conocido. La trayectoria de la compañía Stefanini IT Solutions también confirma el modo gradualista de expansión internacional elaborado por Johanson y Vahlne. En los principios de su actividad en Polonia, la empresa se estableció en unas instalaciones compartidas. Posteriormente, acorde a la ampliación de su actividad, decidió trasladar la oficina hacia una instalación propia para acomodar las necesidades de aumento de plantilla.

\section{CONCLUSIONES}

Este trabajo ha ampliado la literatura relativa al fenómeno de la multinacionalización de las empresas procedentes de mercados emergentes y en cierto modo también la de la inserción en la economía mundial de los países de Europa Central.

Las multilatinas, han aumentado su presencia internacional a lo largo de los últimos años, especialmente desde la primera década del siglo XXI. Como hemos visto, su éxito no radica exclusivamente en una mayor internacionalización sino que se encuentra también en sus posiciones de liderazgo en sus respectivos sectores de actividad. Las empresas de los mercados emergentes que ejecutan su actividad económica en los países desarrollados funcionan como unos delegados económicos de sus países de origen, ofreciendo una imagen fidedigna de su industria y fomentando la confianza hacia sus respectivas economías.

La actual presencia activa de los cinco casos señalados confirma la elevada atracción de la región de la V4 para las empresas que pretenden desarrollar su actividad dentro de la Unión Europea. Los motivos conducentes a esta transnacionalización varían desde las características propias de la geografía de un país en particular, pasando por la cercanía a los grandes mercados europeos, hasta la presencia de una configuración y especialización industrial que fomenta la entrada de empresas dedicadas a un sector industrial determinado. 
Nedela, Daniel. Análisis de la presencia de las multilatinas en el espacio económico del grupo de Visegrád.

Hoy en día tanto por parte del sector privado como por el sector público existe un renovado interés de los países de la V4 y de América Latina en aumentar las relaciones recíprocas de comercio y de inversión. Por parte de los países de la V4, la crisis económica, que azota al mundo occidental desde el año 2007, ha fomentado una mayor demanda por la IED en la Unión Europea como una vía de financiación externa que unida a los crecientes niveles de paro se configura como herramienta útil para el alivio de los presupuestos estatales, tanto en forma de inversión como de creación de empleo. Por el contrario, respecto a los países latinoamericanos, la crisis europea ha creado un entorno que permite a sus empresas acceder a activos en los países desarrollados por un precio inferior debido a la necesidad de financiación de las compañías europeas. A su vez, de este proceso las multilatinas se benefician con un posible trasvase de tecnología y de una posibilidad de financiarse a bajo coste dados los bajos tipos de interés en Europa.

Una posible evolución futura de la presencia de mutilatinas en la región de V4 es difícil de predecir, debido a que los países de las dos regiones mantienen unas relaciones de comercio y de inversión relativamente escasas y muy fluctuantes. La fluctuación de la IED se justifica por medio de una repentina entrada de una empresa al mercado de la V4, en el caso de las multilatinas, o al de América Latina, en el caso de las empresas centroeuropeas.

La amplitud del fenómeno de las multilatinas ofrece varias pistas para una investigación posterior. La V4 es solamente una región de cuatro países de la UE 28, existen varios espacios regionales europeos que podrían ser objeto de una investigación posterior que analizara en detalle los motivos de atracción de multilatinas que están presentes en estos espacios. A su vez sería interesante analizar las estrategias de modo de entrada, las posiciones en el mercado y el volumen de facturación que ostentan estas empresas tanto en las diferentes economías nacionales de la UE como en su conjunto. Por último, tampoco es de menospreciar la actividad gubernamental que se está desarrollando entre las autoridades nacionales, tanto de la V4 como de otros Estados de la UE, con sus homólogos latinoamericanos y el impacto de su actividad en la evolución del comercio y de la IED recíproca. 
Nedela, Daniel. Análisis de la presencia de las multilatinas en el espacio económico del grupo de Visegrád.

\section{BIBLIOGRAFÍA}

Alonso, F. y Revuelta, J. (2013). "Presencia De Multilatinas En Europa. Tipología y Estrategia Empresarial."

Amin, S. (1988). La Desconexión: Hacia Un Sistema Mundial Policéntrico. IEPALA Editorial.

Casanova, L. (2011aㅡ). "El Ascenso De Las Multilatinas En La Economía Mundial." Información Comercial Española, ICE: Revista de economía, (859), 21-31.

. (2011b). "La Década Dorada Latinoamericana: Las" Global Latinas" Llegan a Occidente." Economía exterior: estudios de la revista Política Exterior sobre la internacionalización de la economía española, (59), 85-91.

. (2010). "Las Multinacionales Latinoamericanas En Los Albores De Una Gran Oportunidad." Revista de Administração de Empresas, 50, 439-45.

Casanova, L. et al. (2009). "Multilatinas to Global Latinas: The New Latin American Multinationals."

Casanova, L. y Rodríguez-Montemayor, E. (2014). "The Bridge Model: How Spanish Multinationals Are Building Economic Ties between Asia and Latin America." Business and Politics, 16(3), 373-91.

Cuervo-Cazurra, Á. (2008). "The Multinationalization of Developing Country Mnes: The Case of Multilatinas." Journal of international Management, 14(2), 138-54. 2010. "Multilatinas." Universia business review, (25), 14-33.

Cuervo-Cazurra, Á. y Stal, E. (2011). "The Investment Development Path and Fdi from Developing Countries: The Role of Pro-Market Reforms and Institutional Voids." Latin American Business Review, 12(3), 209-31.

Dunning, J. H. (1994). "Re-Evaluating the Benefits of Foreign Direct Investment." Transnational corporations, 3(1), 23-51.

. (1999). "Trade, Location of Economic Activity and the Multinational Enterprise: A Search for an Eclectic Approach." PJ Buckley \& P. Ghauri. The internationalization of the firm, 61-79.

Fleury, A. (2010). "El Camino Se Hace Al Andar: La Trayectoria De Las Multinacionales Brasileñas." Universia Business Review, (25), 34-55.

Frot, E. y Santiso, J. (2013). "Political Uncertainty and Portfolio Managers in Emerging Economies." Review of International Political Economy, 20(1), 26-51.

Furtado, C. (1974). Teoría y Política Del Desarrollo Económico. Siglo xxi.

Goldstein, A. 1995. "Embraer: From National Champion to Global Player." Financial Times.

Fleury, A et al. (2013). "Differences in Outsourcing Strategies between Firms in Emerging and in Developed Markets." International Journal of Operations \& Production Management, 33(3), 296-321.

Johanson, J. y Vahlne, J. E. (1977). "The Internationalization Process of the Firm-a Model of Knowledge Development and Increasing Foreign Market Commitments." Journal of international business studies, 23-32.

Lessard, D. R. y Reavis, C. (2007). "Globalization 'the Cemex Way,'" MIT Sloan School case,

Lucas, R. E. (1990). "Why Doesn't Capital Flow from Rich to Poor Countries?" The American Economic Review, 92-96.

Nurkse, R. (1952). "Some International Aspects of the Problem of Economic Development." The American economic review, 571-83.

Prebisch, R. (1964). Nueva Política Comercial Para El Desarrollo. Fondo de cultura económica Mexico City. 
Nedela, Daniel. Análisis de la presencia de las multilatinas en el espacio económico del grupo de Visegrád.

Price Waterhouse Coopers (PwC). (2014). "Top 100 Aerospace Companies."

Rivera, R. y Ruelas-Gossi, A. (2007). "Las Multilatinas. Multinacionales Del Nuevo Mundo." Foreign affairs en español, 7(4), 83+-83+.

Rugman, A. M. (2010). "Do We Need a New Theory to Explain Emerging Market Multinationals." Foreign Direct Investment from Emerging Markets: The Challenges Ahead.

. (1981). Inside the Multinationals: The Economics of Internal Markets. Columbia University Press New York.

Santiso, J. (2013). The Decade of the Multilatinas. Cambridge University Press. . 2011. "Los Nuevos Argonautas Latinos." Economía exterior: estudios de la revista Política Exterior sobre la internacionalización de la economía española, (59), 101-08.

Towers Watson. (2010). "Multilatinas Human Capital: Practices Survey." . 2013. "Multilatinas Poised for Growth: Seizing Opportunities in the Global Landscape." 